\title{
Transmucosal Bleomycin for Tongue Lymphatic Malformations
}

\author{
Eric W. Cerrati1, Teresa M. 0², David Binetter ${ }^{2}$, Yelena Bernstein'2, Milton Waner ${ }^{2}$ \\ ${ }^{1}$ Department of Otolaryngology, New York University, New York, USA \\ ${ }^{2}$ Department of Otolaryngology, Vascular Birthmark Institute of New York, Lenox Hill and Manhattan Eye, Ear \\ and Throat Hospitals, New York, USA \\ Email: to@vbiny.org
}

Received 10 February 2015; accepted 28 February 2015; published 4 March 2015

Copyright (C) 2015 by authors and Scientific Research Publishing Inc.

This work is licensed under the Creative Commons Attribution International License (CC BY). http://creativecommons.org/licenses/by/4.0/

(c) (i) Open Access

\begin{abstract}
Purpose: Bleomycin is an antibiotic medication that inhibits the synthesis of DNA, RNA, and proteins and is now used in a variety of medical conditions including vascular anomalies. The aim of this study was to evaluate the clinical efficacy of transmucosal intralesional injection of bleomycin in the management of tongue lymphatic malformations. Method: A single institutional case series was presented on patients with recalcitrant lymphatic malformations of the tongue who were treated with bleomycin. Age at the time of injection, gender, number of treatments, amount of bleomycin injected per session, post-injection complications, pre- and post-injection symptoms, and anatomic extent of the lymphatic malformation were all recorded and analyzed. Results: Five patients received transmucosal bleomycin and were followed over a 10-month period. The patients included 4 females and 1 male, aged from 3.25 to 36 years (average 13.52 years). Four patients had one treatment while 1 required two treatments. A total of 1 to 6 units were injected per session. Overall reduction in size of the lymphatic malformation and improvement in all symptoms were observed in the patients by day 14. Average follow-up was 9 to 12 months. Conclusion: Intralesional injection of bleomycin is an effective treatment modality in patients with lymphatic malformations of the tongue.
\end{abstract}

\section{Keywords}

Bleomycin, Sclerotherapy, Lymphatic Malformation, Tongue

\section{Introduction}

Vascular malformations manifest as either high-flow lesions (arteriovenous malformations and fistulae) or low-

${ }^{*}$ Corresponding author.

How to cite this paper: Cerrati, E.W., O, T.M., Binetter, D., Bernstein, Y. and Waner, M. (2015) Transmucosal Bleomycin for Tongue Lymphatic Malformations. International Journal of Otolaryngology and Head \& Neck Surgery, 4, 81-85.

http://dx.doi.org/10.4236/ijohns.2015.42015 
flow lesions (venous, lymphatic, capillary, or mixed malformations) [1]-[3]. Lymphatic malformations (LMs) are composed of dilated lymphatic vessels with inappropriate drainage patterns and are lined with endothelial cells. They are estimated to occur in $0.5 \%$ of the general population and commonly result in a painless focal mass (macrocystic) or diffuse tissue swelling or overgrowth (microcystic) [2]. While these lesions can occur anywhere on the body, they show a predilection for the cervicofacial region and from a recent review of the anatomic distribution of airway LMs, the most common locations in the upper airway are the oral cavity (75\%), oropharynx (35\%), and the parapharyngeal space (30\%) [4]. LMs involving the tongue tend to be microcystic and poorly defined giving the characteristic granular appearance over the lingual dorsum. Treatment for lymphatic malformations of the tongue is guided by the degree of involvement and the condition of the surrounding structures. Grading systems have been proposed to help standardize treatment; however, these have not been universally accepted. In general, isolated superficial LMs or those confined to only a portion of the tongue allow surgical resection to be an effective treatment. For more advanced LMs, surgical resection alone is inadequate and is often performed in conjunction with other modalities such as laser therapy, radiofrequency ablation, and sclerotherapy.

Currently in non-oral cavity LMs, direct puncture sclerotherapy is used both as an adjunct and as an alternative to surgery [1] [2]. The sclerosants are injected directly into the malformation, causing endothelial damage, inflammation, thrombosis, fibrosis, and eventual destruction of the lesion. The advantages of sclerotherapy include rapid recovery, no incisions, and a low risk of nerve injury and infection [1]. Several different sclerosants have been described in the treatment of LMs. Of these, the most common are sodium tetradecyl sulfate (STS), OK-432, and doxycycline [2]. Unfortunately, most of the sclerosants described in the literature produce a significant amount of postoperative swelling limiting their use when treating airway LMs [1] [2].

Bleomycin, an antibiotic with cytotoxic antitumor properties via the inhibition of DNA, RNA, and protein synthesis, has been used in a variety of medical conditions including in a role as a sclerosant. It is favored by some clinicians because of its clinical efficacy combined with a low incidence of significant postoperative edema [5]-[7]. Bleomycin's use has yet to be applied to oral cavity lymphatic malformations.

\section{Case Report}

A case series of 5 patients with lymphatic malformations of the oral tongue treated with transmucosal bleomycin sclerotherapy between June 2013 and March 2014 is presented. The target lesions were identified preoperatively with computed tomography (CT) and magnetic resonance imaging (MRI). The following inclusion criteria were met by all of the patients: presence of an extensive lymphatic malformation involving the majority $(>75 \%)$ of the anterior tongue; prior treatment consisting of partial $\mathrm{CO}_{2}$ laser ablations; recalcitrant disease with chronic hemorrhagic vesicles, malodor, pain, and swelling; and pre- and post-operative photographs for assessment and comparison. The outcome measures were overall tongue size, number of vesicles, presence of leakage from the vesicles and interval time between treatments.

If and when these patients developed lymphangitis or acute inflammation involving the malformation, treatment consisted of high dose corticosteroids and antibiotics. We recorded the age at the time of the injection, gender, number of treatments, amount of bleomycin injected per session $(0.5 \mathrm{mg} / \mathrm{kg}$ was the maximum dose per session), postinjection complications, pre-/post-injection symptoms and anatomic extent of the LM.

All procedures were performed in the operating room under general anesthesia. A Denhardt mouth gag was used to provide adequate exposure along with either a towel clamp on the distal tongue or a Weider retractor to hold the tongue to one side. A 1-cc syringe with a 25-gauge needle was used. The total amount of bleomycin available per session was $0.5 \mathrm{mg}$ per kilogram with a maximum of $15 \mathrm{mg}$ per session. The needle was advanced across the region of the malformation and filled the malformation with the sclerosant in a retrograde fashion while gradually withdrawing the needle.

The five patients were followed for a period of 9 to 12 months (mean of 10.8) and consisted of four females and one male with ages ranging from 3.25 to 36 years (average 13.52 years). Four of the patients required only one treatment while one required a total of two. A total of 1 to 6 units were injected per session, depending on the patient's weight (Table 1).

All of the patients developed early reactive swelling; however, no airway interventions were necessary. Due to this finding, the injections were limited to the anterior $1 / 2-2 / 3$ of the tongue. In 2 patients, oral corticosteroids were prescribed after 3 - 4 days as the pain from the swelling was affecting their oral intake. At the twoweek follow-up appointment, the swelling was noted to be completely resolved and the overall size of the 
Table 1. Patient data.

\begin{tabular}{cccc}
\hline Patient (age in years, gender) & Location of LM & \# of treatments & Bleomycin amount (units) \\
\hline $36 \mathrm{~F}$ & Anterior tongue, floor of mouth & 1 & 6.0 \\
$3 \mathrm{M}$ & Anterior tongue, floor of mouth & 1 & 1.8 \\
$7 \mathrm{M}$ & Anterior tongue & 1 & 1.7 \\
9F & Anterior tongue & 2 & 6.0 \\
10F & Anterior tongue & 1 & 1.0 \\
\hline
\end{tabular}

tongue was reduced. At the end of the follow-up, the patients reported an overall reduction in tongue size, decreased number of vesicles and increased length of interval time between treatments. The patients also reported an improvement in their quality of life given the effectiveness regarding improvement in speech and reduction/ elimination of hemorrhage, malodor, pain, and swelling.

\section{Discussion}

The treatment for extensive lymphatic malformations of the tongue involves any combination of sclerotherapy, laser therapy, and surgery. Unfortunately, there is no conclusive evidence demonstrating which sclerosant is the best. So far in the literature and with our experience, Bleomycin is the ideal sclerosant for airway lesions because of its effectiveness along with its minimal postoperative swelling [1].

The other sclerotherapy agents such as STS, doxycycline, ethanol and OK-432 have shown varying degrees of effectiveness with significant risk factors. For example, ethanol, though inexpensive and very effective, is associated with nerve injury, skin necrosis and catastrophic systemic effects such as cardiopulmonary collapse. OK-432, an agent once described as being the most widely used sclerosant for LMs, reported a regression of disease in $96 \%$ of patients. Its side effects include swelling, erythema, pain and a low-grade fever for up to 5 days post-injection [2]. Doxycycline has been shown to result in an $83 \%$ mean reduction in lesion size and is deemed more effective in treating microcystic LMs than OK-432. Again, its side effects include pain and significant swelling, which limits its application in airway lesions [3].

Bleomycin was first introduced as a sclerosant in 1977 by Yura et al. It is an antibiotic with cytotoxic antitumoral properties [6]. The agent's low tendency to induce post-injection swelling makes it a preferred sclerosant for airway lesions, especially in patients without tracheotomy at the time of intervention [1] [6] [7]. Our experience has demonstrated a reactive swelling when used specifically in the tongue, which has limited our application of bleomycin to the anterior tongue. This swelling, which was not observed in the supraglottic area specifically, may be secondary to the increased lymphatics and vasculature of the tongue itself [1]. The most worrisome side effect of bleomycin is pulmonary fibrosis. While this complication has been documented in the oncology literature as a dose-dependent response to those who receive the drug systemically, it has never been reported in reference to its use as a sclerosant [8] [9]. The cumulative dose that is associated with the increased risk of pulmonary fibrosis is $450 \mathrm{mg}$ [1]. When used in sclerotherapy, not only is bleomycin not absorbed into the bloodstream but the maximum amount per session is only $15 \mathrm{mg}(0.5 \mathrm{mg} / \mathrm{kg} / \mathrm{session})$, making it extremely unlikely to even approach the high cumulative dose of $450 \mathrm{mg}$ [1] [9].

The more common side effect is hyperpigmentation, which is reported to occur in $8 \%-38 \%$ of patients. This finding does not seem to be dose dependent as it has been reported to occur with doses as low as $5 \mathrm{mg}$. The mechanism of action is unknown; however, histologic studies have shown that bleomycin reduces the epidermal turnover resulting in a prolonged contact between melanocytes and keratinocytes [10]. Local skin irritation after bleomycin results in hyperemia and increased concentration of bleomycin to the area. We, therefore, take the following measures in an effort to reduce the risk of hyperpigmentation: leave all tape including that placed around intravenous lines and EKG leads on the patient until after 72 hours, and advise against scratching the skin. Fortunately, the hyperpigmentation is usually reversible with cessation of the drug but can take up to 6 months to resolve.

Although our case series has only 5 patients, bleomycin has shown promising results in the oral tongue. Once the cohort of patients is increased and the results can be collected on an objective scale, a direct comparison can be made of the different (Figure 1 and Figure 2) treatments such as other sclerosants. Bleomycin has been 


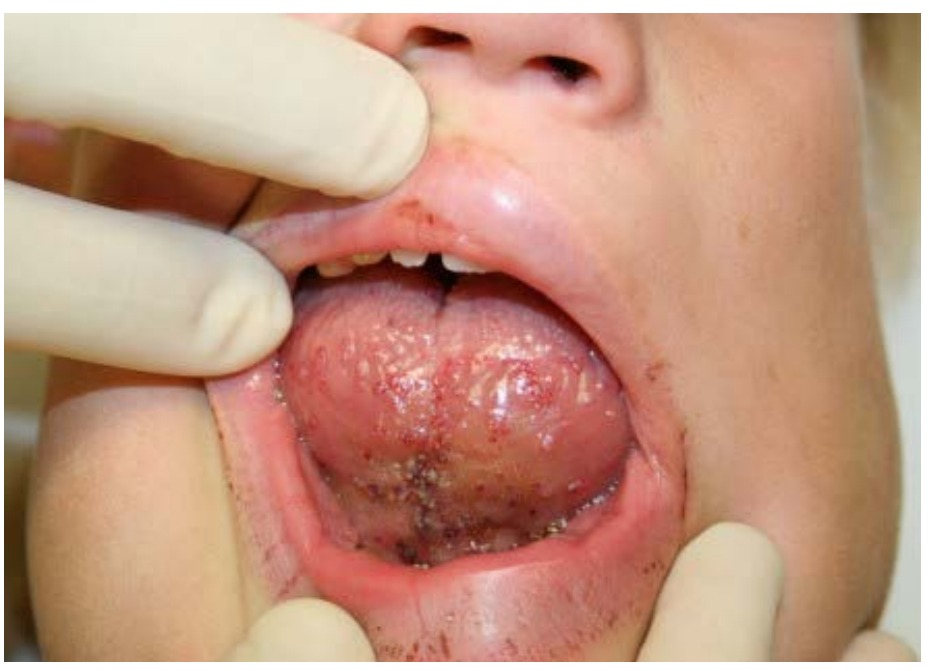

(a)

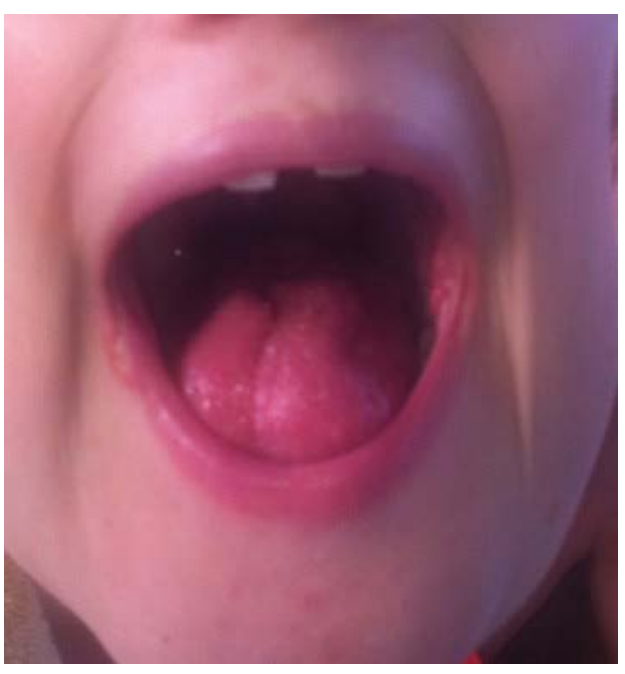

(b)

Figure 1. 10-year-old male with a persistently enlarged tongue despite multiple resections (a); One unit of bleomycin was injected and the follow-up photo was taken 4.5 months later (b).

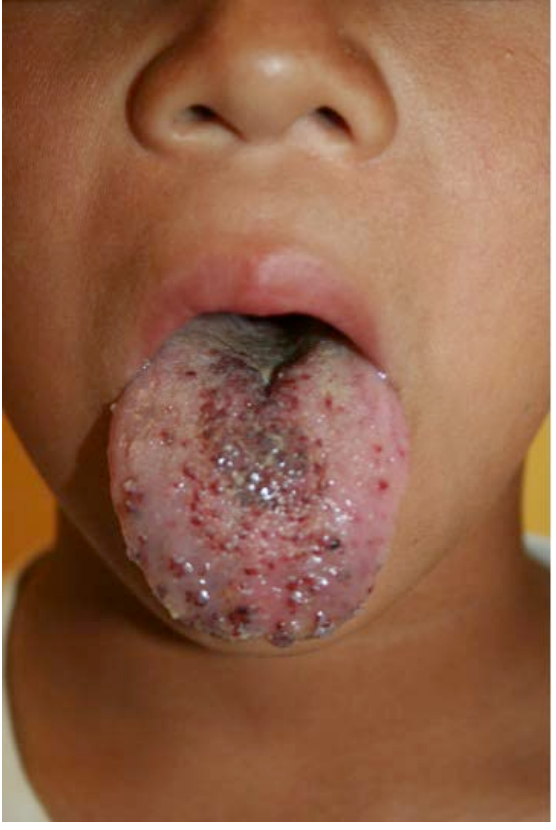

(a)

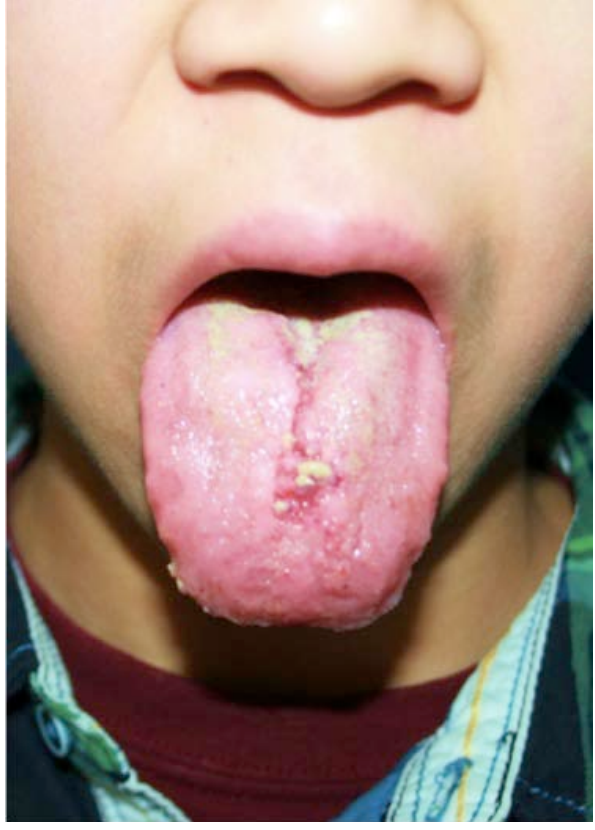

(b)

Figure 2. 7-year-old male demonstrating the classic changes of the tongue after a bleomycin injection (a); At the routine follow-up, the tongue size is reduced and the mucosa has returned to the usual appearance (b).

extremely effective in treating laryngeal LMs and this new application in the tongue will likely be equally successful.

\section{Conclusion}

Intralesional injection of bleomycin is an effective modality of treatment for patients with lymphatic malformations of the tongue. Bleomycin is shown to be an effective sclerosant with a low risk profile. All of the patients in this series were extremely satisfied with the results and no complications or side effects of bleomycin were reported. 


\section{Conflict of Interest}

None.

\section{References}

[1] Oomen, K.P., Paramasivam, S., Waner, M., et al. (2015) Endoscopic Transmucosal Direct Puncture Sclerotherapy for Management of Airway Vascular Malformations. Laryngoscope, Accepted for Publication.

[2] Gurgacz, S., Zamora, L. and Scott, A. (2014) Percutaneous Sclerotherapy for Vascular Malformations: A Systemic Review. Annals of Vascular Surgery, 28, 1335-1349. http://dx.doi.org/10.1016/j.avsg.2014.01.008

[3] Burrows, P.E., Mitri, R.K., Alomari, A., et al. (2008) Percutaneous Sclerotherapy of Lymphatic Malformations with Doxycycline. Lymphatic Research and Biology, 6, 209-216. http://dx.doi.org/10.1089/lrb.2008.1004

[4] O, T.M., Rickert, S.M., Diallo, A.M., et al. (2013) Lymphatic Malformations of the Airway. Otolaryngology-Head and Neck Surgery, 149, 156-160. http://dx.doi.org/10.1177/0194599813485065

[5] Wiegand, S., Eivazi, B., Zimmermann, A., et al. (2009) Microcystic Lymphatic Malformations of the Tongue: Diagnosis, Classification, and Treatment. Archives of Otolaryngology—Head and Neck Surgery, 135, 976-983. http://dx.doi.org/10.1001/archoto.2009.131

[6] Bai, Y., Jia, J., Huang, X.X., et al. (2009) Sclerotherapy of Microcystic Lymphatic Malformations in Oral and Facial Regions. Journal of Oral and Maxillofacial Surgery, 67, 251-256. http://dx.doi.org/10.1016/j.joms.2008.06.046

[7] Mathur, N.N., Rana, I., Bothra, R., et al. (2005) Bleomycin Sclerotherapy in Congenital Lymphatic and Vascular Malformations of Head and Neck. International Journal of Pediatric Otorhinolaryngology, 69, 75-80. http://dx.doi.org/10.1016/j.ijporl.2004.08.008

[8] Jules-Elysee, K. and White, D.A. (1990) Bleomycin-Induced Pulmonary Toxicity. Clinics in Chest Medicine, 11, 1-20.

[9] Ionescu, G., Mabeta, P., Dippenaar, N., et al. (2008) Bleomycin Plasma Spill-Over Levels in Pediatric Patients Undergoing Intralesional Injection for the Treatment of Hemangiomas. South African Medical Journal, 98, 539-540.

[10] Khenaizan, S. and Al-Berouti, B. (2011) Flagellate Pigmentation: A Unique Adverse Side Effect of Bleomycin Therapy. European Journal of Dermatology, 21, 146. 